\title{
Variability of hospital resources for acute care of COPD patients: the European COPD Audit
}

\author{
Jose Luis López-Campos ${ }^{1,2}$, Sylvia Hartl ${ }^{3}$, Francisco Pozo-Rodriguez ${ }^{2,4}$ and \\ C. Michael Roberts ${ }^{5}$ on behalf of the European COPD Audit team
}

Affiliations: ${ }^{1}$ Hospital Universitario Virgen del Rocío, Universidad de Sevilla, Instituto de Biomedicina de Sevilla (IBiS), Seville, ${ }^{2}$ CIBER de Enfermedades Respiratorias (CIBERES), Instituto de Salud Carlos III, Madrid, and ${ }^{4}$ Hospital 12 de Octubre, Instituto de Investigación i+12, Madrid, Spain. ${ }^{3}$ Ludwig Boltzmann Institute of COPD and Respiratory Epidemiology, Vienna, Austria. ${ }^{5}$ Barts and The London School of Medicine and Dentistry, Queen Mary, University of London, London, UK.

Correspondence: J.L. López-Campos, Hospital Universitario Virgen del Rocío, Avda Manuel Siurot, s/n, 41013 Seville, Spain. E-mail: Icamposdasepar.es

ABSTRACT Studies have suggested that larger hospitals have better resources and provide better care than smaller ones. This study aimed to explore the relationship between hospital size, resources, organisation of care and adherence to guidelines.

The European COPD Audit was designed as a pilot study of clinical care and a survey of resources and organisation of care. Data were entered by clinicians to a multilingual web tool and analysed centrally. Participating hospitals were divided into tertiles on the basis of bed numbers and comparisons made of the resources, organisation of care and adherence to guidelines across the three size groups.

13 national societies provided data on 425 hospitals. The mean number of beds per tertile was 220 (lower), 479 (middle), and 989 (upper). Large hospitals were more likely to have resources and increased numbers of staff; hospital performance measures were related in a minority of indicators only. Adherence to guidelines also varied with hospital size, but the differences were small and inconsistent.

There is a wide variation in the size, resources and organisation of care across Europe for hospitals providing chronic obstructive pulmonary disease care. While larger hospitals have more resources, this does not always equate to better accessibility or quality of care for patients.

@ERSpublications

Although large hospitals are more likely to have more resources and staff, hospital performance does not differ greatly http://ow.ly/sfevS

This article has supplementary material available from www.erj.ersjournals.com

Received: April 282013 | Accepted after revision: July 172013 | First published online: Aug 292013

Conflict of interest: Disclosures can be found alongside the online version of this article at www.erj.ersjournals.com

Copyright @ERS 2014 


\section{Introduction}

There is an established body of literature that strongly suggests that larger and more specialised hospital centres provide better care than do smaller units $[1,2]$. There are a number of reasons why this might be the case. For surgical procedures, a higher patient volume may facilitate the growth of greater expertise for the surgeon and also the whole surgical team. The hospital's annual expenditure, the numbers of available doctors or nurse shift patterns are also factors that relate to generic clinical outcomes, suggesting that resources are also important. Recent evidence from the UK indicates that focused organisation of care, as exemplified by stroke, heart attack and trauma centres, where units are designed to care for patients with a specific presenting condition, also provides better patient outcomes [2-4].

Chronic obstructive pulmonary disease (COPD) is a highly prevalent condition, generating large numbers of admissions throughout Europe, with a high risk of short- and long-term all-cause mortality [5]. A single small study from the UK demonstrated that small district general hospitals had higher COPD patient mortality than did larger and university hospitals, and were less likely to provide optimum process of care. More detailed analysis showed this to be related to the numbers of available medical staff [6]. Similar wide variations in the provision of resources for the treatment of in-hospital COPD have been shown in Spain [7].

Although there are European and international guidelines on the management of COPD, there are no guidelines that recommend minimum resources or suggest how services should be organised to deliver optimum care. In this study, we explore the variation in the provision of resources to manage patients admitted with COPD exacerbation across 13 European countries, and the association between the size of the hospitals and the provision of resources, looking at the relationship between resources and clinical care provision. This article is the report from the organisation and resources survey, and will be followed by further detailed clinical analyses in due course.

\section{Methods}

The methodology of the European COPD Audit has been extensively described elsewhere [8]. Briefly, this European Respiratory Society (ERS)-funded COPD audit was designed as a pilot study to evaluate clinical practice variability as well as clinical and organisational factors related to outcomes for COPD hospital admissions across Europe. It was designed as a prospective, observational, noninterventional cohort trial. The study comprised a first 8-week phase, during which all consecutive cases admitted to hospital due to an exacerbation of COPD were identified and information on clinical practice was gathered. During this period, participating hospitals were also asked to complete a cross-sectional survey of the resources and organisation devoted to COPD acute care. The present article describes the results of the hospital resources and organisation of the participant hospitals across Europe.

The ERS managed the audit and named a steering committee to oversee the process, which reported to the ERS Executive Committee. Items for the organisational database were selected by the steering committee, based upon those that had previously been used and validated in the Spanish and UK national audits [7, 9]. The survey included questions related to the hospital and to the respiratory unit resources separately. An item list of the final survey is available [8]. A number of terms had to be consensually defined, as there was no shared meaning across Europe, and were considered key concepts for the audit, as follows.

Ward: clinical area in which patients are nursed in beds as admissions to hospital.

Respiratory department: an integrated clinical grouping of healthcare workers (clinicians and administrators) whose responsibility is to care for patients with respiratory conditions. This excludes healthcare workers whose responsibilities are purely research without a clinical role. A department may function within one hospital or across more than one hospital.

Hospital: a healthcare facility located in a particular geographical site. It may comprise one or several buildings, but these buildings are administered by a single executive board.

Unit: a functional healthcare facility that is often identical to a hospital, but may include more than one hospital and/or more than one geographical location. However, the unit functions as a single administrative and healthcare facility. Examples may include two, or even more, hospitals that previously were independent but have since merged clinical and administrative functions.

A software company (IDCode, Lausanne, Switzerland) was commissioned to design a web-based collection tool encompassing both the organisation and clinical databases. Data were entered remotely at each participating site to a centrally controlled server. The web tool was established as a multilingual database, to allow the data to be documented in the language of each participating country. The web tool provided a help facility with explanatory text to assist with interpretation of the survey questions. 
In order to study the relationship between hospital resources and the clinical care provided, 10 key clinical statements from the Global Initiative for Chronic Obstructive Lung Disease (GOLD) 2010 strategy (contemporary with the audit period) regarding clinical care provided during an admission were identified. Adherence to these statements using an analysis of the clinical dataset was compared across the three types of centre.

\section{Statistical analysis}

Statistical computations were performed using the Statistical Package for Social Sciences (SPSS, version 20.0; IBM Corporation, Somers, NY, USA). In order to compare hospitals, these were divided into tertiles according to their size as measured by the number of beds across all participant hospitals. Thus, hospitals were categorised into small, medium or large according to the 33rd and 66th percentiles in the number of beds. All items were characterised by the European mean value and the intercountry range (ICR), indicating those countries with the lowest and highest values for each item. In the tables, the number of centres that provided information is provided for each variable. Comparisons between countries were evaluated by Chisquared test and ANOVA. $\alpha$-error was set at 0.05 .

\section{Results}

Of the 432 participating hospitals, five centres in the UK, one in Belgium and one in Slovakia did not complete the organisational survey, resulting in 425 (98.4\%) recording information for the present analysis. The distribution of hospitals in the different participant countries and the proportion of each national population covered by those hospitals are given in figure 1 . The number of beds varied between 40 and 3800 ; the 33rd percentile was 367 beds and the 66th percentile was 600 beds.

\section{Hospital resources}

The description of the hospital resources is summarised in table 1 . The great majority of participant centres were public around half of them university or teaching hospitals. Spirometry was readily available in most centres. In $79.9 \%$ of centres, there was both a respiratory ward and respiratory team, whereas $8.7 \%$ did not have either of these resources.

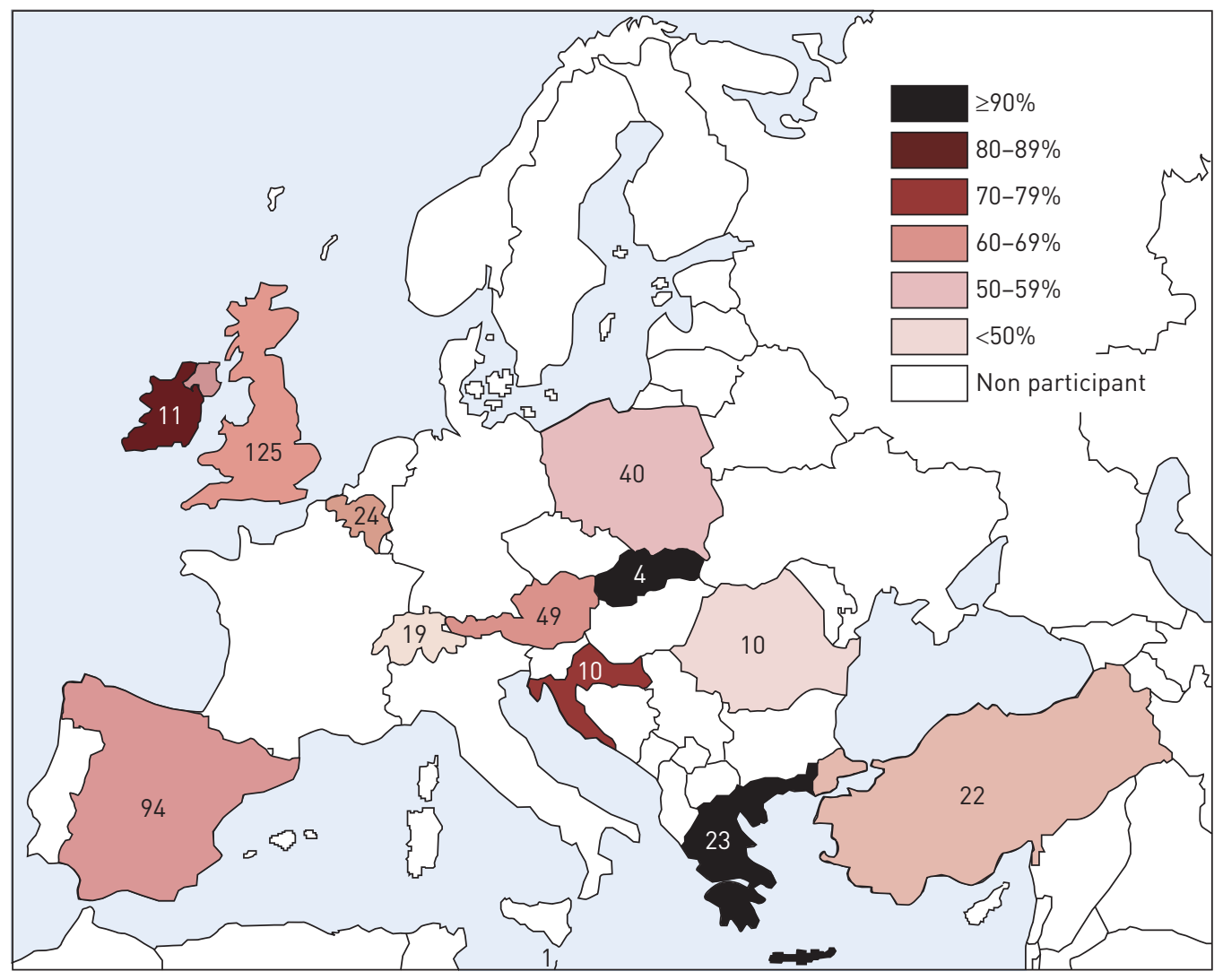

FIGURE 1 Distribution of participant hospitals and proportion of population screened. 185814623 (62.8\%) patients were screened. 
TABLE 1 Characteristics of the participant hospitals in the European COPD Audit

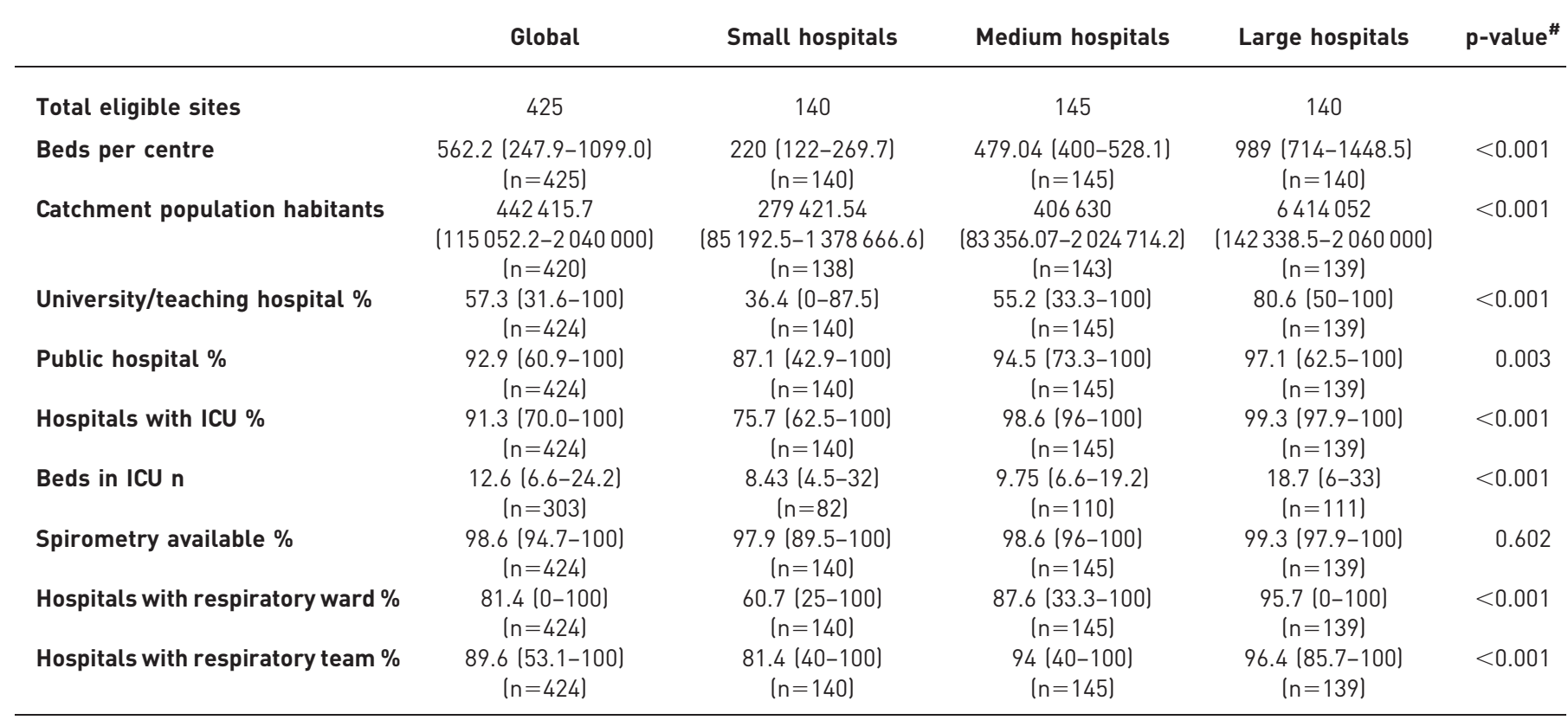

Data are presented as $\mathrm{n}$ or mean (intercountry range), unless otherwise stated. COPD: chronic obstructive pulmonary disease; ICU: intensive care unit. " : calculated by Chi-squared test or ANOVA, as appropriate.

\section{Respiratory unit resources}

The resources of the respiratory units are summarised in table 2. There was great variation in the provision of outpatient clinics, with a lower number for those specifically designed for COPD patients, regardless of hospital size. The presence and size of high-dependency units were also highly variable in the participant countries, but were present in a minority overall, except in the UK. Although a majority of centres offered invasive and noninvasive ventilation, quite a number of centres declared not to have the capacity to ventilate all eligible patients. Rehabilitation programmes were of limited availability in most countries. Only Belgium, Ireland, Switzerland and the UK had a majority of hospitals $(\sim 80 \%)$ offering this service. The most frequent type of rehabilitation programme was hospital based (35\%, ICR 0-66.7\%), whereas 30.5\% (ICR $0-100 \%$ ) of centres provided both hospital- and home-based rehabilitation programmes, and $15.8 \%$

TABLE 2 Resources of the participant units in the European COPD Audit

\section{Global}

Units $\mathrm{n} / \mathrm{N}$

Respiratory outpatient clinic \%

COPD outpatient clinic \%

Respiratory ward \%

Admission ward for COPD \%

System of specialty triage \%

Emergency department \%

High-dependency unit \%

Beds in the high-dependency unit $\mathbf{n}$

Offer noninvasive ventilation for acidotic patients $\%$

Offer invasive ventilation for acidotic patients $\%$

Access to respiratory rehabilitation programmes \%

Access to palliative care service $\%$
$422 / 425$

$\begin{array}{cc}422 / 425 & 139 / 140 \\ 90.0(44.9-100) & 82.7(30-100) \\ 61.8(0-82.1) & 50.4(15-90) \\ 78.9(0-100) & 60.4(25-100) \\ 65.4(26.5-100) & 51.1(16.7-100) \\ 32.0(0-100) & 25.2(0-100) \\ 80.1(50-100) & 68.3(21.1-100) \\ 49.3(10-100) & 45.3(0-100) \\ 7.1(2.0-11.2) & 5.9(2-9) \\ 89.6(60-100) & 84.2(62.5-100) \\ 75.8(33.3-100) & 66.2(28.6-100) \\ 50.0(0-90.9) & 41.7(10-92.3) \\ 59.7(4.5-100) & 52.5(0-100)\end{array}$

Small hospitals Medium hospitals
Large hospitals $\mathbf{p}$-value ${ }^{\#}$

$139 / 140$

$95.7(78.6-100) \quad 0.001$

$73.4(0-100) \quad<0.001$

$93.5(0-100)<0.001$

$72.7(25-100) \quad<0.001$

$38.1(0-100) \quad 0.067$

$86.3(50-100)<0.001$

$57.6(0-100) \quad 0.059$

$8.2(3.3-19) \quad 0.061$

$97.1(75-100) \quad 0.001$

$79.9(0-100) \quad 0.005$

$60.4(0-100) \quad 0.006$

$63.3(0-100) \quad 0.107$

Data are presented as mean (intercountry range), unless otherwise stated. COPD: chronic obstructive pulmonary disease. ${ }^{\#}$ : Chi-squared test or ANOVA, as appropriate. 
TABLE 3 Staffing of the respiratory units surveyed

\begin{tabular}{|c|c|c|c|c|c|}
\hline & Global & Small hospitals & Medium hospitals & Large hospitals & p-value ${ }^{\#}$ \\
\hline Units $n / N$ & $422 / 425$ & $139 / 140$ & $144 / 145$ & $139 / 140$ & \\
\hline \multicolumn{6}{|c|}{ Respiratory specialists } \\
\hline n per 1000 beds & $14.7(7.05-74.3)$ & $22.1(7.5-88.9)$ & $12.8(3.9-80.0)$ & $9.4(4.8-14.7)$ & $<0.001$ \\
\hline \multicolumn{6}{|c|}{ Respiratory trainees } \\
\hline $\mathrm{n}$ & $3.7(1.3-9.6)$ & $2.04(0.4-10.6)$ & $3.3(0.8-13.2)$ & $5.9(2.4-9)$ & $<0.001$ \\
\hline $\mathrm{n}$ & $2.1(0.6-6)$ & $1.6(0.3-3.4)$ & $2.01(0-13.2)$ & $2.5(0.6-6.5)$ & 0.021 \\
\hline $\mathrm{n}$ per 1000 beds & $5.05(1.6-20.0)$ & $8.3(2.4-20.0)$ & $4.1(0-26.2)$ & $2.6(0.6-8.0)$ & $<0.001$ \\
\hline \multicolumn{6}{|l|}{ Nurse specialists } \\
\hline $\mathrm{n}$ & $7.9(0-63.6)$ & $5.9(0-69)$ & $11.4(0-140)$ & $6.2(0-31.5)$ & 0.245 \\
\hline $\mathrm{n}$ per 1000 beds & $20.3(0.0-272)$ & $31.5(0-321.9)$ & $23.0(0.0-467.5)$ & $6.5(0.0-21.1)$ & 0.017 \\
\hline \multicolumn{6}{|c|}{ Lung function technicians } \\
\hline
\end{tabular}

Data are presented as mean (intercountry range), unless otherwise stated. ${ }^{\#}$ : Chi-squared test or ANOVA, as appropriate.

TABLE 4 Organisational performance of the respiratory units

\section{Global}

Small hospitals
Medium hospitals

$22547.6(886.6-61438.4) \quad 13866.8(250-42272.8)$

$$
(n=341)
$$

$60.6(51.4-96)$

$(\mathrm{n}=280)$

$71.9(35.1-100)$

$(n=424)$

$49.5(18.2-100)$

$(n=424)$

$1.8(1.0-4.8)$ $(\mathrm{n}=401)$

Ward rounds by admitting

specialist $\mathbf{n}$

Patients seen by

physiotherapist \%

Patients seen by

respiratory specialist \%

Capacity to noninvasively

ventilate all eligible

patients \%

Capacity to invasively ventilate

all eligible patients $\%$

Early supported discharge

programme \%

Admissions that enter the early

discharge programme $\%$

Takes care of long-term

oxygen therapy \%

Takes care of home

mechanical ventilation \%

Eligible patients who receive

pulmonary rehabilitation $\%$
$53.2(21.5-100)$ ( $n=384$ )

$69.3(44.8-100)$ $(\mathrm{n}=388)$

$67.5(0-90.9)$ ( $n=378$ )

$71.6(0-100)$

$(\mathrm{n}=320)$

$32(0-75.2)$

( $n=422$ )

$37.3(15-90)$

$(n=110)$

$87.4(63.3-100)$ ( $n=422$ )

$59.5(20.0-100)$ ( $n=422)$

$42.1(17.5-80.0)$ $(n=132)$
( $n=116$ )

66.4 (47-95.4) $(n=70)$

$59.3(30.3-100)$ $(n=140)$

45 (9.5-100) ( $n=140)$

$1.6(1.2-2.8)$ ( $n=132$ )

$49.1(14.4-100)$ $(\mathrm{n}=111)$

64.7 (34-100) ( $n=131)$

66.7 (33.3-100) $(n=117)$

$69.6(33.3-100)$ $(\mathrm{n}=92)$

$15.8(0-60)$ $(n=139)$

$45.7(20-73.3)$ ( $\mathrm{n}=19$ )

$84.9(47.4-100)$ ( $n=139)$

56.1 (10.5-84.8) $(n=139)$

$42.6(10-55)$
145

Medium ho

20

$2107.1(1113.7-49735.6)$
$(n=112)$
$57.8(37.4-100)$
$(n=97)$
$75.9(33.3-100)$
$(n=145)$
$41.4(0-100)$
$(n=145)$
$1.5(1.2-2.3)$
$(n=134)$

$51.1(21.4-91.2)$ $(\mathrm{n}=115)$

$66.9(28.6-100)$ ( $n=127)$

$68.3(0-100)$ ( $n=126$ )
$69.2(0-100)$ $(\mathrm{n}=117)$
$36.8(0-100)$ $(n=144)$
$36.8(5-90)$ $(\mathrm{n}=43)$

86.8 (40-100) ( $n=144)$

$53.5(0-100)$ ( $n=144)$

$48.9(20-80)$ $(n=40)$

Data are presented as n or mean lintercountry rangel, unit. ${ }^{\#}$ : Chi-squared test or ANOVA, as appropriate. 


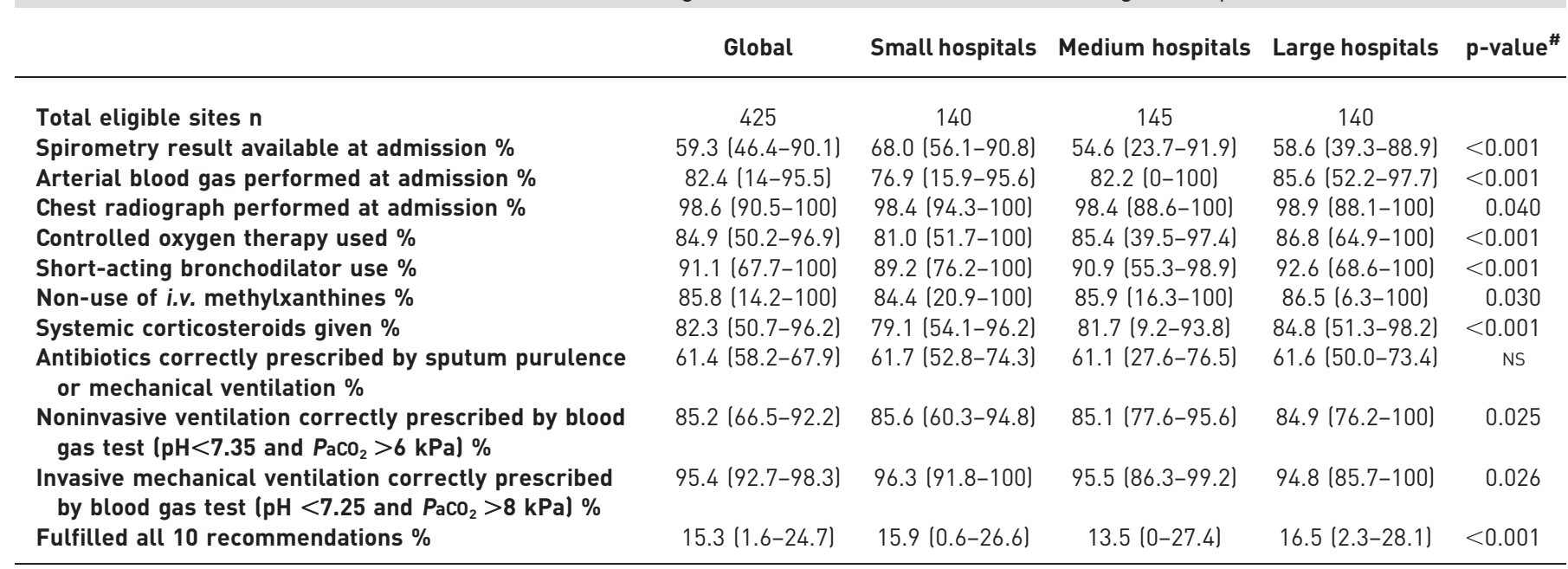

Data are presented as mean (intercountry range), unless otherwise stated. $\mathrm{PaCO}_{2}$ : arterial carbon dioxide tension; NS: nonsignificant. ${ }^{\#}$ : Chi-squared test.

(ICR 0-76.5\%) provided only home-based rehabilitation programmes. A minority of centres (18.7\%, ICR 0-33.3\%) declared the provision of "other", nonspecified types of rehabilitation programmes.

Staffing levels are summarised in table 3. Once again, great variation between hospitals can be observed, with larger hospitals tending to have more staff, although there is a significant overlap between the categories of hospital bed size. However, in the case of nurse specialists, medium sized hospitals were reported to have more of this grade of staff than both large and small hospitals.

Information regarding the organisational performance of the respiratory units is summarised in table 4 . While in some cases, the larger hospitals had better organisational performance, such as a respiratory physician on call every day, this was not consistently the case. For example, larger hospitals had more specialist staff and were more likely to have a specialist respiratory ward, but were no more likely than smaller hospitals to triage COPD patients into those specialist services (table 3). In a similar fashion, larger hospitals were more likely to offer noninvasive ventilation but were no more likely to have the capacity to ventilate all appropriate patients.

The association between GOLD 2010 statements adherence and the hospital size is summarised in table 5 . Although there were statistical differences in adherence to most of the GOLD recommendations, the difference between the three types of hospitals were not very consistently relevant. Some recommendations improved with hospital size, such as arterial blood gas on admission, controlled oxygen therapy use, shortacting bronchodilator use, non-use of methylxanthines or systemic corticosteroid use. On the contrary, some recommendations were more frequently followed by small hospitals, for example spirometry results available, or mechanical ventilation use. Others remained unrelated to hospital size, like chest radiographs and antibiotics use.

\section{Discussion}

The present study describes for the first time the resources available for healthcare provision to COPD patients admitted to hospital in 13 European countries. The results of the organisational information from the European COPD Audit show a great variability in different aspects between the different sizes of hospitals and between countries. Although large hospitals have more resources, this does not imply better access to services or a striking difference in adherence to guidelines. The present data provide a broad picture that may help healthcare managers to identify those areas of improvement needed in each location and brings an opportunity to set some minimum standards across Europe for providing clinical care to COPD patients.

The main strength of this study is the novel information it provides and a wide coverage in the catchment population screened, as well as the methodology of auditing different healthcare systems in different languages [8]. However, in order to interpret our results, a number of issues must be considered. This was a pilot study and, as such, did not include all European countries. Within each country, the participating 
hospitals volunteered, so there is no attempt at representative sampling. There is a lack of consistency across Europe in the titles and roles of staff, and in the definitions of key organisation and resource elements of the audit. For instance, the concept of a nurse specialist or a specialty triage system may not have the same meaning in different countries. To reduce this interpretation bias, some concepts were specifically defined, and there were explanatory tags in the web tool, to help researchers incorporate the requested information [8]. The different number of participating hospitals per country was another key factor. The number of hospitals per country ranged from one (Malta) to 125 (UK), largely related to the size of the country. The statistical effect is that the countries contributing smaller numbers of hospitals result in higher intercountry variation. In the same way, some correlations between hospital size and performance may be affected by the practice across all hospital sizes in one of the larger national contributors to the dataset. Despite these limitations, we believe that this dataset represents the largest available comparative survey of European hospitals treating COPD patients. While the results should be interpreted in the context of the limitations defined above, there is much valuable information to be analysed.

Larger hospitals tend to have greater resources than do smaller ones. In some cases, this also allows better provision of services. However, both the availability of services to patients and the adherence to management recommendations are no better among large hospitals than it is among smaller ones. It may be that for the majority of COPD patients, smaller hospitals provide equally good care as larger ones. Providing care in a local setting, which is integrated to local services, may be an advantage in many cases. Larger hospitals with higher numbers of specialists may offer advantages to a minority of patients who need tertiary care interventions, but these audit data suggest that there is little difference in the care quality offered on average. This suggests that either greater resources are matched to greater workload, or that resources are not organised in an efficient manner. Indeed, table 3 suggests that although larger hospitals have more staff, the ratio of staff to beds is lower than in smaller hospitals. Such a situation may mitigate against specialists caring for every COPD patient admitted, but should still provide opportunities for better organisation of specialist care. For example, a specialty on-call rota or the staffing of a respiratory ward.

If we view these findings from the perspective of the patient, the variability is likely to cause some concern. The European Union has benefitted large populations by the introduction of Europe-wide standards, yet there are no standards for the provision of a hospital service. This raises the question of what the minimum acceptable standards should be to provide safe, high-quality care for patients. The findings of this audit present an opportunity for the medical profession and others dedicated to the provision of high-quality care to patients across Europe to initiate a process of defining minimum standards for units providing services to such patients.

There is particular concern where strongly evidence-based services are not available across a wider range of hospitals and countries. The evidence about the efficacy and cost-effectiveness of intermediate-care units is strong [10], but the provision of these types of units was low in the majority of countries. It is interesting that quite a number of centres declared not to have the capacity to treat all eligible patients throughout the year for invasive and noninvasive ventilation. It is well known that COPD exacerbations cluster in time, with an increase in colder months [11] and in certain geographical areas [12]. This variability provides an organisational challenge, as the available ventilators and staff may also need to change during the year, but it is a challenge that is not met in over a fifth of hospitals (table 4). The use of home mechanical ventilation was not in the scope of the present study and has been evaluated in a previous European survey [13].

Pulmonary rehabilitation improves survival in those patients who survive a hospital admission due to COPD [14] as well as a number of other clinically relevant outcomes, either after or during the admission [15]. Nonetheless, despite this demonstrated effect, the provision of rehabilitation is far from optimal in Europe. In this context, where resources are scarce, the implementation of home- or community-based rehabilitation programmes may be a more cost-effective solution that is demonstrated to be effective in providing rehabilitation programmes in the community setting [16]. Another, more simple approach would be promoting daily physical exercise in the community, known to have positive impact on health status [17].

Early supported discharge programmes are also known to be safe and effective, reducing time in hospital without an adverse impact on mortality, but were also not frequently used. In a recent randomised controlled trial, an early supported discharge programme with community nurses resulted in a feasible alternative to traditional hospital admission [18].

Palliative care services included a broad group of services for either in-hospital or out-patient palliative care. Severe acute exacerbations of COPD are associated with deterioration in lung function and represent one of the most important adverse prognostic factors [19]. Frequently, the management of COPD exacerbations requires urgent medical care and hospitalisation. Unfortunately, several patients with acute exacerbations of COPD die during the hospital admission [20] or within the months following hospital discharge [21]. 
Symptom management is one of the most important aspects of end-of-life care, because COPD symptoms often worsen in the final days. In this context, the availability of palliative care units offers the opportunity to meet the needs of patients and their carers towards the end of life [22].

Staff provision has recently been described to have an impact on the outcomes of admitted patients [3]; however, the relationship between hospital staffing and access to resources or adjustment to guidelines has not been described. The present data document the number of professionals and trainees available in the participant units. In this regard a European regulation to define the staff provision for a specific service is lacking and needed.

In summary, the present study describes the resources available and the organisation of care provided by individual hospitals and units in 13 European countries in relation to COPD admissions. This study has shown huge variation in both the resourcing and the organisation of care amongst European hospitals caring for acute COPD admissions. This variability applies both between countries and within countries. Further prospective audits, mapping patient outcomes to resource provision and service organisation may inform the development of pan-European recommendations. This information will help managers and policy makers to evaluate the provision of resources in their own country, to adapt these to the necessities of COPD patients admitted to hospital, and to result in improving this care and avoiding inequalities in European health systems.

\section{Acknowledgements}

The authors would like to thank all the contributing clinicians across Europe and to the statistical team of A. CastroAcosta (Hospital 12 de Octubre, Instituto de Investigación i+12, Madrid, Spain), V. Abraira-Santos (Hospital Universitario Ramón y Cajal, IRYCIS, Centre for Biomedical Research on Epidemiology and Public Health (CIBERESP), Instituto de Salud Carlos III, Madrid, Spain), A. López-Quilez (Dept of Statistics and Operational Research, Universidad de Castellón, Castellón, Spain) and J. Dorado (PERTICA, Spain).

The European COPD Audit team members are as follows. Steering committee: C.M. Roberts, S. Hartl and J.L. LópezCampos. Data analysis team: F. Pozo-Rodríguez, J.L. López-Campos, A. Castro-Acosta, V. Abraira-Santos, A. LópezQuilez and J. Dorado. National experts (by alphabetical order of country): O. Burghuber and R. Kohansal (Austria); W. Janssens and V. Heinen (Belgium); N. Miculinic and H. Puretic (Croatia); N. Tzanakis and E. Nontas Kosmas (Greece); S. McCormack and T. McDonell (Ireland); C. Farrugia Jones (Malta); J. Chorostowska-Wynimko (Poland); F. Mihaltan, M. Alexandru Bogdan and I. Munteanu (Romania); I. Solovic and R. Tkacova (Slovakia); F. Pozo-Rodríguez and J. Ancochea (Spain); D. Stolz (Switzerland); M. Polatli and E. Sen (Turkey); C. Bucknall, S. Welham and C. Routh (UK). Project managers: M. Haan, M. Zarelli, E. Lechat and R.J. Buckingham. ERS COPD Audit liaison officer: G. Joos. For a complete list of participants and affiliations see the online supplementary material.

\section{References}

1 Stukel TA, Fisher ES, Alter DA, et al. Association of hospital spending intensity with mortality and readmission rates in Ontario hospitals. JAMA 2012; 307: 1037-1045.

2 Romley JA, Jena AB, Goldman DP. Hospital spending and inpatient mortality: evidence from California: an observational study. Ann Intern Med 2011; 154: 160-167.

3 Needleman J, Buerhaus P, Pankratz VS, et al. Nurse staffing and inpatient hospital mortality. N Engl J Med 2011; 364: $1037-1045$.

4 Griffiths P, Murrells T, Dawoud D, et al. Hospital admissions for asthma, diabetes and COPD: is there an association with practice nurse staffing? A cross sectional study using routinely collected data. BMC Health Serv Res 2010; 10: 276.

5 Diaz-Guzman E, Khosravi M, Mannino DM. Asthma, chronic obstructive pulmonary disease, and mortality in the U.S. population. COPD 2011; 8: 400-407.

6 Roberts CM, Barnes S, Lowe D, et al. Evidence for a link between mortality in acute COPD and hospital type and resources. Thorax 2003; 58: 947-949.

7 Pozo-Rodríguez F, López-Campos JL, Alvarez-Martinez CJ, et al. Clinical audit of COPD patients requiring hospital admissions in Spain: AUDIPOC study. PLoS One 2012; 7: e42156.

8 López-Campos JL, Hartl S, Pozo-Rodríguez F, et al. European COPD Audit: design, organisation of work and methodology. Eur Respir J 2013; 41: 270-276.

9 Roberts CM, Lowe D, Bucknall CE, et al. Clinical audit indicators of outcome following admission to hospital with acute exacerbation of chronic obstructive pulmonary disease. Thorax 2002; 57: 137-141.

10 Scala R. Respiratory high-dependency care units for the burden of acute respiratory failure. Eur J Intern Med 2012; 23: 302-308

11 Jenkins CR, Celli B, Anderson JA, et al. Seasonality and determinants of moderate and severe COPD exacerbations in the TORCH study. Eur Respir J 2012; 39: 38-45.

12 Holt JB, Zhang X, Presley-Cantrell L, et al. Geographic disparities in chronic obstructive pulmonary disease (COPD) hospitalization among Medicare beneficiaries in the United States. Int J Chron Obstruct Pulmon Dis 2011; 6: 321-328.

13 Lloyd-Owen SJ, Donaldson GC, Ambrosino N, et al. Patterns of home mechanical ventilation use in Europe: results from the Eurovent survey. Eur Respir J 2005; 25: 1025-1031.

14 Puhan MA, Gimeno-Santos E, Scharplatz M, et al. Pulmonary rehabilitation following exacerbations of chronic obstructive pulmonary disease. Cochrane Database Syst Rev 2011; 10: CD005305. 
15 Reid WD, Yamabayashi C, Goodridge D, et al. Exercise prescription for hospitalized people with chronic obstructive pulmonary disease and comorbidities: a synthesis of systematic reviews. Int J Chron Obstruct Pulmon Dis 2012; 7: 297-320.

16 Moore L, Hogg L, White P. Acceptability and feasibility of pulmonary rehabilitation for COPD: a community qualitative study. Prim Care Respir J 2012; 21: 419-424.

17 Orrow G, Kinmonth AL, Sanderson S, et al. Effectiveness of physical activity promotion based in primary care: systematic review and meta-analysis of randomised controlled trials. BMJ 2012; 344: e1389.

18 Utens CM, Goossens LM, Smeenk FW, et al. Early assisted discharge with generic community nursing for chronic obstructive pulmonary disease exacerbations: results of a randomised controlled trial. BMJ Open 2012; 2: e001684.

19 Soler-Cataluña JJ, Martínez-García MA, Román Sánchez P, et al. Severe acute exacerbations and mortality in patients with chronic obstructive pulmonary disease. Thorax 2005; 60: 925-931.

20 Kinnunen T, Säynäjäkangas O, Keistinen T. Features of hospitalisations for acute exacerbation of COPD resulting in death. Monaldi Arch Chest Dis 2007; 67: 10-14.

21 Kinnunen T, Säynäjäkangas O, Keistinen T. The COPD-induced hospitalization burden from first admission to death. Respir Med 2007; 101: 294-299.

22 Curtis JR. Palliative and end-of-life care for patients with severe COPD. Eur Respir J 2008; 32: 796-803. 